\title{
Controversies in extracorporeal membrane oxygenation (ECMO) utilization and congenital diaphragmatic hernia (CDH) repair using a Delphi approach: from the American Pediatric Surgical Association Critical Care Committee (APSA-CCC)
}

\author{
Sarah B. Cairo ${ }^{1}$ - Mary Arbuthnot ${ }^{2} \cdot$ Laura A. Boomer $^{3} \cdot$ Michael W. Dingeldein $^{4} \cdot$ Alexander Feliz $^{5} \cdot$ Samir Gadepalli $^{6}$. \\ Chris R. Newton ${ }^{7}$. Robert Ricca Jr. ${ }^{2} \cdot$ Adam M. Vogel $^{8}$. David H. Rothstein ${ }^{1,9}$. On behalf of the American Pediatric \\ Surgical Association, Surgical Critical Care Committee
}

Accepted: 10 August 2018 / Published online: 21 August 2018

(c) Springer-Verlag GmbH Germany, part of Springer Nature 2018

\begin{abstract}
Purpose Review current practices and expert opinions on contraindications to extracorporeal membrane oxygenation (ECMO) in congenital diaphragmatic hernia (CDH) and contraindications to repair of CDH following initiation of ECMO. Methods Modified Delphi method was employed to achieve consensus among members of the American Pediatric Surgical Association Critical Care Committee (APSA-CCC).

Results Overall response rate was $81 \%$ including current and former members of the APSA-CCC. An average of 5-15 CDH repairs were reported annually per institution; $26-50 \%$ of patients required ECMO. $100 \%$ of respondents would not offer ECMO to a patient with a complex or unrepairable cardiac defects or lethal chromosomal abnormality; $94.1 \%$ would not in the setting of severe intracranial hemorrhage (ICH). $76.5 \%$ and $72.2 \%$ of respondents would not offer CDH repair to patients on ECMO with grade III-IV ICH or new diagnosis of lethal genetic or metabolic abnormalities, respectively. There was significant variability in whether or not to repair $\mathrm{CDH}$ if unable to wean from ECMO at 4-5 weeks.

Conclusions Significant variability in practice pattern and opinions exist regarding contraindications to ECMO and when to offer repair of $\mathrm{CDH}$ for patients on ECMO. Ongoing work to evaluate outcomes is needed to standardize management and minimize potentially futile interventions.
\end{abstract}

Level of evidence $\mathrm{V}$ (expert opinion).

Keywords Congenital diaphragmatic hernia $\cdot$ Extracorporeal membrane oxygenation $\cdot$ ECMO $\cdot \mathrm{CDH} \cdot$ Pediatric surgery

Electronic supplementary material The online version of this article (https://doi.org/10.1007/s00383-018-4337-y) contains supplementary material, which is available to authorized users.

Sarah B. Cairo

scairo2@gmail.com

1 Department of Pediatric Surgery, John R. Oishei Children's Hospital, 1001 Main Street, Buffalo, NY 14203, USA

2 Department of Pediatric and General Surgery, Naval Medical Center Portsmouth, Portsmouth, USA

3 Department of Surgery, Children's Hospital of Richmond at Virginia Commonwealth University, Richmond, USA

4 Department of Pediatric Surgery, Rainbow Babies and Children Hospital, Cleveland, USA

5 Department of Pediatric Surgery, University of Tennessee Health Sciences, Memphis, USA
6 C.S. Mott Children's Hospital, Michigan Medicine, Ann Arbor, USA

7 Department of Pediatric Surgery, University of California at San Francisco Benioff Children's Hospital Oakland, San Francisco, USA

8 Department of Pediatric General Surgery, Texas Children's Hospital, Houston, USA

9 Department of Surgery, Jacobs School of Medicine, State University of New York, University at Buffalo, Buffalo, USA 


\section{Introduction}

Congenital diaphragmatic hernia $(\mathrm{CDH})$ is a common congenital birth defect occurring at an incidence of approximately 1 in 5000 births [1]. There have been significant improvements in survival and increased variability in management strategies over the past several decades in patients with $\mathrm{CDH}$ [2]. Current estimates for overall survival of patients born with $\mathrm{CDH}$ range from 8 to $80 \%$ [3, 4]. Improvement in survival is widely thought to be associated with changes in ventilator strategies, management of pulmonary hypertension, improvements in surgical technique and anesthesia, and the use of extracorporeal membrane oxygenation (ECMO) when indicated [5].

The use of ECMO for patients with $\mathrm{CDH}$ was first reported in 1977. Despite several technological advances and studies on its utility, consensus regarding comprehensive management are lacking. For other common conditions requiring ECMO such as meconium aspiration, congenital cardiac defects, and cardiomyopathy with or without cardiogenic shock, outcomes based on duration of ECMO and anticipated outcomes have been evaluated [6-8]. It requires a multidisciplinary team to manage neonates on ECMO, thus, variability in management is inevitable. Timing of ECMO initiation and timing of surgical repair with regard to ECMO have been addressed with significant variability based on surgeon and anesthesiologist comfort and preference, institutional practices, and surgeon training $[9,10]$.

The primary objectives of this study were to review current practices and expert opinions on two areas particularly underrepresented in the literature regarding the use of ECMO for CDH: situations in which ECMO should not be offered to patients with $\mathrm{CDH}$; and situations in which repair of $\mathrm{CDH}$ should not be offered after placing a patient on ECMO. We hypothesized that despite gathering a group of experts in the field with substantial combined, and independent, experience with neonatal ECMO, there would be areas of controversy with regard to the specific scenarios addressed.

\section{Methods}

Prior to study initiation, the protocol was reviewed by the University at Buffalo Institutional Review Board and found to be exempt from full review (STUDY00001754).

To address these controversial topics in the management of $\mathrm{CDH}$, a three-step modified Delphi method was employed [11]. The process involved a qualitative assessment, a ranking evaluation, and a consensus assessment.
Given the paucity of literature on the topics of interest, the Delphi method, a consensus-based technique, was utilized for collecting and aggregating informed judgments from a group of experts via multiple iterations. This method was used to maximize the benefits of an expert panel while maintaining anonymity.

\section{Participant selection}

The surveys at each step of the Delphi method were distributed to members of the American Pediatric Surgical Association Critical Care Committee (APSA-CCC). Participating committee members are all board certified in pediatric surgery and surgical critical care. Additionally, the majority of committee members are directors of surgical critical care fellowship training programs and/or medical directors of surgical intensive care units. All participants practice within the United States or Canada, with some variability in prior research experience, clinical focus, and career stage.

\section{Delphi procedure}

\section{Phase I: qualitative assessment}

The first phase used a web-based survey to ask participants a series of open-ended and ranking questions relevant to the use of ECMO in patients with CDH (Supplement 1). Participants were invited to complete the survey via email and were asked to answer to the best of their ability as a reflection of their current practice patterns.

\section{Phase II: ranking evaluation}

De-identified responses from the first stage of the Delphi method were reviewed by a core group of committee members. Similar ideas and opinions related to the use of ECMO for $\mathrm{CDH}$ were clustered together into opinion statements. Specifically, the second stage included questions regarding contraindications to placing a patient with $\mathrm{CDH}$ on ECMO and when repair of the $\mathrm{CDH}$ should not be offered after placing a patient on ECMO (Supplement 2). Participants were asked to rank each statement on a 5-point Likert scale based on their level of agreement with the statement.

Based on responses to open-ended questions and multiple-choice questions in phase I of the Delphi approach, ten items were selected for inclusion as absolute contraindications to offering ECMO to a patient with $\mathrm{CDH}$. These statements were reviewed by a core group of committee members and compared to published guidelines on the use of ECMO for $\mathrm{CDH}$ and other neonatal conditions. Responses and comments to the remainder of items were reviewed and slight modifications to statements were made for inclusion in the third portion of the Delphi approach. For example, instead of 
a gestational age less than 32 weeks as an absolute contraindication, a gestational age less than 30 weeks was included for Phase III.

\section{Phase III: consensus assessment}

Following completion of the second stage, responses were again reviewed and consensus or variation evaluated. For statements with high levels of variability (low levels of consensus), literature was reviewed and distributed to the participants who were encouraged to complete independent review as needed. Based on literature review and review by core group of committee members, a revised ranking evaluation was distributed including open-ended questions to encourage anonymous modification of initial responses based on other members' views (Supplement 3).

\section{Statistical analysis}

Data were summarized and descriptive statistics based on committee member responses included. High levels of agreement or consensus were obtained for four items as determined by greater than $50 \%$ of respondents selecting "agree" or "strongly agree". All data analysis was performed with Microsoft Excel (2010) and IBM SPSS Statistics 24 (2016). Significance was accepted at $p<0.05$. Intraclass correlation coefficients (ICC) were also calculated to evaluate for changes in reliability across the different phases of the Delphi approach. Reliability measurements were calculated for absolute agreement between multiple raters for each concept.

\section{Results}

\section{Phase I: qualitative assessment}

Eighteen members of the APSA-CCC completed the survey at each stage of the Delphi process. Respondent characteristics were assessed in the first survey (Table 1). 16 unique institutions were represented with participants from current (75\%) and immediate past (25\%) CCC. More than $50 \%$ of participants reported managing $5-15$ patients with $\mathrm{CDH}$ per year. $44 \%$ of respondents reported placing 26-50\% of patients with CDH on ECMO. One respondent reported that their institution did not use ECMO and was excluded from remainder of analysis. Of the participants who used $\mathrm{ECMO}$ for $\mathrm{CDH}$ at their institution, the majority reported management by an interdisciplinary team including neonatologists or pediatric intensivists and pediatric surgeons. There was significant variability regarding timing of repair and type of repair (thoracoscopic versus

Table 2 Indications for ECMO

\begin{tabular}{ll}
\hline Factor & $\%$ respondents \\
\hline Instability of preductal saturations & 75.0 \\
Preductal saturations $<85 \%$ in first $24 \mathrm{~h}$ & 31.3 \\
Following addition of pressors & 25.0 \\
Following trial of nitric oxide & 25.0 \\
Following trial of steroids & 6.3 \\
Following trial of intravenous pulmonary vasodila- & 6.3 \\
$\quad$ tors & \\
First blood gas after birth & 6.3 \\
Oxygenation index $>40$ & 56.3 \\
Oxygenation index 25-40 & 43.8 \\
Persistent, refractory acidosis & 12.5 \\
\hline
\end{tabular}

Table 1 Survey response to controversial topics in the use of extracorporeal membrane oxygenation in patients with congenital diaphragmatic hernia, $n(\%)$

\begin{tabular}{|c|c|c|c|}
\hline & $\begin{array}{l}\text { Disagree/strongly } \\
\text { disagree }\end{array}$ & Agree/strongly agree & $\% \mathrm{CV}$ \\
\hline \multicolumn{4}{|l|}{ a. Contraindications to ECMO } \\
\hline Complex, unrepairable cardiac defect & $0(0)$ & $18(100)$ & 70.2 \\
\hline Lethal chromosomal abnormality & $0(0)$ & $18(100)$ & 55.4 \\
\hline Gestational age $<32$ weeks & $7(38.9)$ & $6(33.3)$ & 237.4 \\
\hline Intracranial hemorrhage grade III or IV & $0(0)$ & $16(88.9)$ & 87.8 \\
\hline \multicolumn{4}{|c|}{ b. Situations where CDH repair should not be offered after ECMO initiated } \\
\hline Occurrence of massive intracranial hemorrhage while on ECMO & $1(5.6)$ & $15(83.3)$ & 98.7 \\
\hline Newly diagnosed lethal genetic or metabolic abnormality & $0(0)$ & $17(94.4)$ & 78.0 \\
\hline Unable to come off ECMO after 4-5 weeks & $6(33.3)$ & $8(44.4)$ & 215.1 \\
\hline
\end{tabular}


open) for patients not on ECMO, although all respondents reported open repair when on ECMO.

Regarding indications for ECMO, the most frequently cited factors included instability of preductal saturations, preductal saturations $<85 \%$ in the first $24 \mathrm{~h}$, and high oxygenation index ( $>40$ ) (Table 2). Open-ended questions were included to identify situations in which ECMO should not be offered to a patient with a CDH. A variety of responses were obtained including primarily patient characteristics such as birth weight, gestational age, presence of intracerebral hemorrhage and associated anomalies. The most common cited contraindications to ECMO were birth weight, gestational age, grade III-IV intracranial hemorrhage, significant coagulopathy or uncontrolled bleeding and major cardiac malformation were cited most commonly (Table 3). When decisions were made regarding repair of $\mathrm{CDH}$ and use of ECMO, most respondents described a multidisciplinary approach, with pediatric specific palliative care available to $75 \%$ of respondents.

\section{Phase Il: ranking evaluation}

Three items were identified for inclusion as situations where $\mathrm{CDH}$ repair should not be offered to a patient after placing them on ECMO. Two items were identified as having high levels of agreement (occurrence of a massive intracranial hemorrhage while on ECMO and new diagnosis of a lethal genetic or metabolic abnormality). The remaining item, inability to come off ECMO within 4-5 weeks, had significant variability in responses. A comprehensive literature review was performed to identify studies addressing outcomes and maximum time on ECMO. Articles were distributed to committee members for review (Supplement 4) prior to Phase III of the Delphi approach. Intraclass correlation coefficient to assess inter-rater reliability was 0.93 (95\% CI 0.87-0.97). When separated by topic, intraclass correlation was highest, with the narrowest confidence interval, for level of agreement with contraindications to ECMO (ICC 0.94, 95\% CI 0.88-0.98) compared to situations where $\mathrm{CDH}$ repair should not be offered (ICC $0.94,95 \%$ CI $0.75-1.00$ ) and indications for ECMO (ICC 0.86, 95\% CI 0.64-0.98).
Table 3 Contraindications for ECMO

\begin{tabular}{lc}
\hline Factor & \% respondents \\
\hline Birthweight <2000 gm & 62.5 \\
Gestational age $<34$ weeks & 43.8 \\
Intracranial hemorrhage grade I & 6.3 \\
Intracranial hemorrhage grade II & 43.8 \\
Intracranial hemorrhage grade III & 75.0 \\
Intracranial hemorrhage grade IV & 81.3 \\
Significant coagulopathy or uncontrolled bleeding & 56.3 \\
Mechanical ventilation > 10-14 days & 25.0 \\
Major cardiac malformation & 56.3 \\
Severe pulmonary hypoplasia (predicted lung volume <15\%) & 37.5 \\
Oxygenation index <40 & 12.5 \\
\hline
\end{tabular}

Table 4 Survey response to controversial topics in the use of extracorporeal membrane oxygenation in patients with congenital diaphragmatic hernia (round 3 Delphi approach), $n(\%)$

\begin{tabular}{|c|c|c|c|}
\hline & $\begin{array}{l}\text { Disagree/strongly } \\
\text { disagree }\end{array}$ & Neutral & Agree/strongly agree \\
\hline \multicolumn{4}{|l|}{ a. Contraindications to ECMO } \\
\hline Complex, unrepairable cardiac defect & $0(0)$ & $0(0)$ & $18(100)$ \\
\hline Lethal chromosomal abnormality & $0(0)$ & $0(0)$ & $18(100)$ \\
\hline Gestational age $<30$ weeks & $2(11.1)$ & $6(33.3)$ & $10(55.6)$ \\
\hline Intracranial hemorrhage grade III or IV & $1(5.9)$ & $0(0)$ & $16(94.1)$ \\
\hline \multicolumn{4}{|c|}{ b. Situations where CDH repair should not be offered after ECMO initiated } \\
\hline Occurrence of massive intracranial hemorrhage while on ECMO & $2(11.1)$ & $2(11.1)$ & $15(76.5)$ \\
\hline Newly diagnosed lethal genetic or metabolic abnormality & $1(5.6)$ & $4(22.2)$ & $13(72.2)$ \\
\hline Unable to come off ECMO after $4-5$ weeks & $9(50.0)$ & $4(22.2)$ & $5(27.8)$ \\
\hline
\end{tabular}




\section{Phase III: consensus assessment}

A third and final survey was distributed to committee members after review of responses and literature. A total of 19 committee members responded to this survey, all of whom had completed the first two portions of the Delphi process. With modifications based on the second phase, a high level of agreement was achieved for six items on the list of contraindications to ECMO in a patient with $\mathrm{CDH}$ and again, two items on the list of situations where $\mathrm{CDH}$ repair should not be offered after placing a patient on ECMO (Table 4). A telephone conference call was also conducted including survey respondents to review the results of the survey. The points of greatest discordance were discussed along with a review of available literature specific to these controversial topics. Intraclass correlation coefficient to assess inter-rater reliability was essentially unchanged overall when compared to Phase IIat 0.93 (95\% CI 0.86-0.98). When separated by topic of concern, higher intraclass correlation was noted for level of agreement with contraindications to ECMO (ICC 0.95, 95\% CI 0.87-0.99) compared to situations where $\mathrm{CDH}$ repair should not be offered (ICC 0.89, 95\% CI 0.56-1.00).

\section{Discussion}

Based on the results of this study, it is clear that despite the advances in medical and surgical care, there remains variable agreement between providers on how best to address controversial and multifaceted management plans in neonates with $\mathrm{CDH}$ requiring ECMO. $\mathrm{CDH}$ is a complex and often times fatal defect of development. Despite a high incidence and movement towards centralization of management to highvolume centers, there is significant variability in management $[12,13]$. Two areas of particular interest, situations where ECMO should not be offered to a patient with $\mathrm{CDH}$ and situations where repair of $\mathrm{CDH}$ should not be offered to a patient placed on ECMO were addressed in this study. Given the variability in management identified on studies by the Children's Hospitals Neonatal Consortium CDH Study Group and review of data collected by the Extracorporeal Life Support Organization (ELSO), a Delphi approach was employed to achieve consensus among pediatric surgeons of the APSA-CCC committee $[1,14,15]$.

With the increasing utilization of ECMO for patients with $\mathrm{CDH}$ and other pathology, several institutions have put forth guidelines regarding indications, contraindications, and protocols for appropriate use. In a systematic review published by the APSA Outcomes and Evidence Based Practice Committee in 2015, 21 papers were selected for in-depth review regarding ECMO utilization in $\mathrm{CDH}$ [3]. Though other areas of $\mathrm{CDH}$ management including preferred mode and support of the guidelines utilized by the University of Michigan
(Supplement 4) are outlined in this review, contraindications to ECMO cannulation were excluded [16-18].

\section{Contraindications to initiation of ECMO}

This study sought to identify specific contraindications for ECMO in patients with $\mathrm{CDH}$ but found several factors achieving variable levels of agreement among committee members. High levels of agreement were found for patients with complex, unrepairable cardiac defects, lethal chromosomal abnormalities, intracranial hemorrhage grade III-IV, and pre-existing diagnosis of significant coagulopathy or bleeding disorder. These factors are all found to be independently associated with higher risk of mortality in neonates and increased rates of complications specific to ECMO and otherwise impaired life expectancy. In a survey of active neonatal ECMO centers, greater than $90 \%$ of respondents would not offer ECMO to a neonate with Trisomy 13 or 18, while $73 \%$ would not offer ECMO to a patient with grade III or IV intraventricular hemorrhage [19, 20]. Pre-existing intracranial hemorrhage has long been considered a relative contraindication for ECMO based on the need for systemic anticoagulation [21]. Advances in ECMO technology and introduction of novel anticoagulants, however, have allowed for use of ECMO in the setting of traumatic brain injury and stroke though further data is needed before ECMO use in the setting of high grade ICH would be recommended in pediatric patients [22]. While presence of pre-existing bleeding disorder and coagulopathy were identified as a contraindication for ECMO by greater than $50 \%$ of respondents in the first phase of this study, more recent literature demonstrates no difference in intracranial hemorrhage or infarct in pediatric ECMO patients based on coagulation profile [23].

Two factors frequently cited in the literature as barriers to cannulation for ECMO and receiving a significant amount of attention in this study include gestational age and weight. In a review of factors affecting outcomes on ECMO, Bokman et al. reported disparities in outcome and resource utilization in pediatric patients [6]. For patients specifically with $\mathrm{CDH}$, variable results are reported based on gestational age and weight at time of cannulation [24]. Birthweight $<2 \mathrm{~kg}$ is frequently cited as cutpoint for higher risk of mortality though gestational age is a bit more variable [25]. Data recently published from the ELSO registry, adjusted for comorbidities and complications, however, demonstrated no change in mortality based on birthweight. Consistent with previous literature, gestational age rather than birthweight was found to be independently associated with increased mortality [26, 27]. Though there was significant discordance in the present study on whether or not gestational age and birthweight should be recommended as contraindications to ECMO in $\mathrm{CDH}$, agreement improved when weight limit was decreased to $<1800 \mathrm{~g}$ and gestational age to $<30$ weeks. 


\section{Contraindications to $\mathrm{CDH}$ repair after initiation of ECMO}

The second point of emphasis of this study identifies situations in which a patient has already been placed on ECMO but repair of the $\mathrm{CDH}$ may be controversial or even contraindicated. Potential scenarios where this may arise were introduced by committee members and set forth to the group through the surveys. Three factors were included in the final phase of the Delphi process with again variable levels of agreement. The two points reaching highest consensus are the development of a massive head bleed (intracranial hemorrhage grade III or IV) or diagnosis with a lethal genetic or metabolic abnormality while on ECMO, two variables previously cited as contraindications to ECMO if identified prior to cannulation.

Unfortunately, consensus on the optimal duration of ECMO or time after which $\mathrm{CDH}$ repair should not be offered, was not achieved through the Delphi process. Many respondents described protocols to determine when repair was to be performed, if done on ECMO, with the majority occurring within the first $48 \mathrm{~h}$ of cannulation. Other respondents reported inability to come of ECMO as an indication for repair of $\mathrm{CDH}$. Of note, in all circumstances, respondents described a multidisciplinary team approach to the decision not to offer repair of CDH. Very few facilities had neonatal specific palliative care teams but the majority had designated providers for the pediatric services.

This ambiguity is consistent with the current literature. Pugliandla et al. concluded that while no specific lengths of runs of ECMO can be recommended, acceptable survival was observed up to 4 weeks and that some patients may even require a second trial of ECMO [4, 28, 29]. In a review of the pediatric ELSO registry from 2009 to 2015, the average run duration for patients with $\mathrm{CDH}$ was 12 days with $50 \%$ survival to hospital discharge compared to meconium aspiration, the second most common respiratory diagnosis, with average run duration of 6 days and $93 \%$ survival to discharge [15]. Additional retrospective reviews demonstrated longer durations of ECMO for patients repaired "late" on ECMO (average $18 \pm 6.1$ days) compared to "early" repair on ECMO (average 12 \pm 7.5 days) and following decannulation (average $10 \pm 3.5$ days), but are unable to fully account for intrinsic differences in patients deemed stable enough for early versus late repair or decannulation [30, 31].

\section{Limitations}

One limitation of using the Delphi approach to establish consensus is in the limited number of participants in the survey process. While the study included only members of the APSA-CCC, a committee whose primary goal is to represent the APSA on issues such as these, members of the surgical community with high-volume experience and expertise specifically regarding ECMO and $\mathrm{CDH}$, were not included. Many members of the committee chair their respective critical care departments and oversee fellowship training and were appointed committee membership based on election and is felt to represent a large proportion of the pediatric surgical community.

While surveys and Delphi processes may be limited by group think or over representation of the opinions of a few outspoken individuals, this survey was completed anonymously without sharing of rolling results. Individuals could only complete the survey once for each round of the study thus ensuring uniform representation among participants. To that end, studies of this nature are limited in the support of large databases and randomized controlled studies. They do, however, provide a foundation for moving forward with such studies based on clinical experience, expertise, and equipoise.

Finally, there are many areas of controversy with regards to the management of ECMO in patients with $\mathrm{CDH}$. These two questions were selected for review based largely on the paucity of literature to address them and the frequency with which they present in clinical practice. Other questions for consideration, on which significantly more literature has become available in the past two decades, such as percutaneous versus open access for ECMO and timing of repair of $\mathrm{CDH}$ with respect to ECMO decannulation, were not addressed in this manuscript. They do, however, warrant ongoing discussion amongst experts in the field.

\section{Conclusions}

Despite the limitations inherent to the methods employed, this study successfully addressed two controversial topics in the management of patients with $\mathrm{CDH}$. Based on the consensus of the APSA Critical Care Committee, it is our recommendation that (1) ECMO not be offered to patients with complex, unrepairable cardiac defects, lethal chromosomal abnormalities, intracranial hemorrhage grade III-IV, and pre-existing diagnoses of significant coagulopathy or bleeding; and (2) for patients already on ECMO, a multidisciplinary team should be involved in the decision of whether or not to offer repair of $\mathrm{CDH}$ to patients diagnosed with a new, high-grade intracranial hemorrhage, patients with a new diagnosis of lethal genetic or metabolic disorder, and, depending on institutional policies, to those unable to make significant progress after an extended duration of time on ECMO. We recommend institution-specific protocols for facilitating the care of complex neonates to include duration of ECMO and timing of repair (level V evidence, expert opinion) with ongoing data collection for standardized outcome assessment. 
Funding This research did not receive any specific Grant from funding agencies in the public, commercial, not-for-profit sectors.

\section{Compliance with ethical standards}

Conflict of interest Dr. Cairo reports that she has no conflict of interest. Dr. Arbuthnot reports that she has no conflicts of interest. Dr. Boomer has no conflicts of interest to report. Dr. Dingeldein has no relevant conflicts of interest to report. Dr. Feliz has no conflicts of interest to report. Dr. Gadepalli reports that he has no conflicts of interest. Dr. Newton reports that he has no conflicts of interest. Dr. Ricca Jr has no conflicts of interest to report. Dr. Vogel reports no conflicts of interest relevant to the present study. Senior author, Dr. Rothstein, reports that he has no conflicts of interest.

Ethical approval All procedures performed in studies involving human participants were in accordance with the ethical standards or the institutional and/or national research committee and with the 1964 Helsinki Declaration and its later amendments or comparable ethical standards.

\section{References}

1. Morini F, Goldman A, Pierro A (2006) Extracorporeal membrane oxygenation in infants with congenital diaphragmatic hernia: a systematic review of the evidence. Eur J Pediatr Surg 16(6):385-391

2. Kays DW et al (2013) Long-term maturation of congenital diaphragmatic hernia treatment results: toward development of a severity-specific treatment algorithm. Ann Surg 258(4):638-644 (discussion 644-5)

3. Puligandla PS et al (2015) Management of congenital diaphragmatic hernia: a systematic review from the APSA outcomes and evidence based practice committee. J Pediatr Surg 50(11):1958-1970

4. Seetharamaiah R et al (2009) Factors associated with survival in infants with congenital diaphragmatic hernia requiring extracorporeal membrane oxygenation: a report from the Congenital Diaphragmatic Hernia Study Group. J Pediatr Surg 44(7):1315-1321

5. Keijzer R, Puri P (2010) Congenital diaphragmatic hernia. Semin Pediatr Surg 19(3):180-185

6. Bokman CL et al (2015) Determinants of survival and resource utilization for pediatric extracorporeal membrane oxygenation in the United States 1997-2009. J Pediatr Surg 50(5):809-814

7. Paden ML et al (2014) Update and outcomes in extracorporeal life support. Semin Perinatol 38(2):65-70

8. Ryan CA et al (1994) Extracorporeal membrane oxygenation in infants with congenital diaphragmatic hernia and cardiac malformations. J Pediatr Surg 29(7):878-881

9. Vaja $\mathrm{R}$ et al (2017) The use of extracorporeal membrane oxygenation in neonates with severe congenital diaphragmatic hernia: a 26-year experience from a tertiary centre $\dagger$. Eur J Cardiothorac Surg 52:552-557

10. Bryner BS et al (2009) Congenital diaphragmatic hernia requiring extracorporeal membrane oxygenation: does timing of repair matter? J Pediatr Surg 44(6):1165-1171 (discussion 1171-2)

11. Hsu C-C, Sandford BA (2007) The Delphi technique: making sense of consensus. Practical Assess Res Eval 12(10):1-8

12. Grushka JR et al (2009) Effect of hospital case volume on outcome in congenital diaphragmatic hernia: the experience of the Canadian Pediatric Surgery Network. J Pediatr Surg 44(5):873-876

13. Salazar JH et al (2016) Regionalization of pediatric surgery: trends already underway. Ann Surg 263(6):1062-1066

14. Morini $\mathrm{F}$ et al (2017) Treatment strategies for congenital diaphragmatic hernia: change sometimes comes bearing gifts. Front Pediatr 5:195

15. Barbaro RP et al (2017) Pediatric Extracorporeal Life Support Organization registry international report 2016. ASAIO J 63(4):456-463

16. Kays DW et al (2015) Outcomes in the physiologically most severe congenital diaphragmatic hernia $(\mathrm{CDH})$ patients: whom should we treat? J Pediatr Surg 50(6):893-897

17. Guner YS et al (2009) Outcome analysis of neonates with congenital diaphragmatic hernia treated with venovenous vs venoarterial extracorporeal membrane oxygenation. J Pediatr Surg 44(9):1691-1701

18. Dimmitt RA et al (2001) Venoarterial versus venovenous extracorporeal membrane oxygenation in congenital diaphragmatic hernia: the Extracorporeal Life Support Organization Registry, 1990-1999. J Pediatr Surg 36(8):1199-1204

19. Chapman RL et al (2009) Patient selection for neonatal extracorporeal membrane oxygenation: beyond severity of illness. J Perinatol 29(9):606-611

20. Kuo KW et al (2017) Should extracorporeal membrane oxygenation be offered? An international survey. J Pediatr 182:107-113

21. Biscotti $\mathrm{M}$ et al (2015) Extracorporeal membrane oxygenation use in patients with traumatic brain injury. Perfusion 30(5):407-409

22. Cho HJ et al (2017) Anticoagulation therapy during extracorporeal membrane oxygenator support in pediatric patients. Chonnam Med J 53(2):110-117

23. Anton-Martin P et al (2017) Coagulation profile is not a predictor of acute cerebrovascular events in pediatric extracorporeal membrane oxygenation patients. ASAIO J Pediatr Circulatory Support 63:793-801

24. Botden SM et al (2017) Bilateral congenital diaphragmatic hernia: prognostic evaluation of a large international cohort. J Pediatr Surg 52(9):1475-1479

25. Delaplain PT et al (2017) Cannulating the contraindicated: effect of low birth weight on mortality in neonates with congenital diaphragmatic hernia on extracorporeal membrane oxygenation. J Pediatr Surg 52:2018-2025

26. Harting MT, Lally KP (2014) The Congenital Diaphragmatic Hernia Study Group registry update. Semin Fetal Neonatal Med 19(6):370-375

27. Stevens TP et al (2002) Survival in early- and late-term infants with congenital diaphragmatic hernia treated with extracorporeal membrane oxygenation. Pediatrics 110(3):590-596

28. Kays DW et al (2014) Extracorporeal life support in patients with congenital diaphragmatic hernia: how long should we treat? J Am Coll Surg 218(4):808-817

29. Tiruvoipati $\mathrm{R}$ et al (2007) Predictors of outcome in patients with congenital diaphragmatic hernia requiring extracorporeal membrane oxygenation. J Pediatr Surg 42(8):1345-1350

30. Fallon SC et al (2013) Repair of congenital diaphragmatic hernias on Extracorporeal Membrane Oxygenation (ECMO): does early repair improve patient survival? J Pediatr Surg 48(6):1172-1176

31. Karimova A et al (2009) Neonatal extracorporeal membrane oxygenation: practice patterns and predictors of outcome in the UK. Arch Dis Child Fetal Neonatal Ed 94(2):F129-F132 